\title{
Can peripheral blood monocyte percentage and lymphocyte monocyte ratio at diagnosis predict survival in pediatric neuroblastoma patients?
}

\author{
Koray Yalçı1 ${ }^{1 \oplus}$, Gülen Tüysüz ${ }^{1 \oplus}$, Funda Tayfun Küpesiz ${ }^{1 \oplus}$, Selen Bozkurt² ${ }^{2 \oplus}$, \\ Alphan Küpesiz ${ }^{1 \oplus}$, Elif Güler ${ }^{1 \oplus}$ \\ Departments of ${ }^{1}$ Pediatric Hematology/Oncology and BMT Unit and ${ }^{2}$ Biostatistics and Medical Informatics, Akdeniz University \\ Faculty of Medicine, Antalya, Turkey.
}

\begin{abstract}
Background. Previous studies have shown that the immune system plays a critical role in cancer pathogenesis. The lymphocyte monocyte ratio (LMR) and monocyte percentage (MP) have been found to be prognostic factors in various types of adult cancers. But studies about pediatric tumors are scarce and to our knowledge, there are no studies evaluating the immune system effect in pediatric neuroblastoma patients. The aim of this study was to assess whether LMR and MP at diagnosis may have an effect on prognosis in neuroblastoma patients.
\end{abstract}

Methods. We retrospectively analyzed MP and LMR at diagnosis in 71 pediatric neuroblastoma patients treated between 2002 and 2016.

Results. The optimal cut-off values of LMR and MP were determined using the receiver operating characteristics curves (ROC) and area under the curve (AUC). We found that a low LMR $(\leq 3.5)$ and a high MP $(\geq 7.5 \%)$ were correlated with worse overall survival and shorter event-free survival in univariate analysis. Multivariate analysis revealed that elevated LMR was an independent factor for better OS and EFS.

Conclusions. In conclusion, LMR and MP might be valuable prognostic factors for predicting OS in neuroblastoma patients. Multicenter and prospective studies are warranted to confirm this hypothesis.

Key words: neuroblastoma, lymphocyte monocyte ratio, monocyte percentage, immune system.

The immune system has an important role on the outcome of cancer patients. It can prevent tumor outgrowth or conversely immune cells can help tumor outgrowth. Recent studies have shown that advanced cancer patients had a low lymphocyte count and this was associated with poor overall survival in various cancer types. ${ }^{1-4}$ Monocytes also have a crucial role in tumor response. Inflammatory monocytes in peripheral blood are recruited by certain chemokines into the tumor microenvironment where they differentiate into tumor associated macrophages and promote angiogenesis,

$\bowtie \quad$ Elif Güler

elifguler@akdeniz.edu.tr

Received 18th November 2020, revised 21st January 2021, accepted 22nd January 2021. metastasis, immune suppression and chemo resistance. ${ }^{5}$ The prognostic value of lymphocyte to monocyte ratio (LMR) has been investigated in hematologic malignancies and a low LMR was reported as an unfavorable prognostic factor in patients with diffuse large B cell lymphoma and Hodgkin lymphoma. ${ }^{6,7}$ There are also numerous studies regarding the prognostic role of LMR in solid tumors such as soft tissue sarcomas, nasopharyngeal carcinoma, and ovarian cancer but all of these studies comprise only of adult patients. To our knowledge there is no study investigating the prognostic value of LMR and peripheral blood monocyte count in any childhood cancer.

Neuroblastoma is the most common extra cranial solid tumor of childhood and the most frequently diagnosed cancer in infancy. ${ }^{8,9}$ 
Neuroblastoma has a diverse pattern of clinical presentation; the disease course ranges from spontaneous regression to aggressive metastatic tumor. ${ }^{10}$ It is regarded as one of the most common cancers that undergo spontaneous regression. ${ }^{11-13}$ The induction of patients' immune response toward their own tumor cells is one of the mechanisms suggested to contribute to this phenomenon.

Age, stage, histology and genetic changes (ploidy, MYCN copy number, 11q deletion) are the well-known prognostic factors in neuroblastoma. Patients are stratified into risk groups and treated according to these prognostic factors. In this study, we aimed to analyze whether peripheral LMR and blood monocyte percentage (MP) might have an effect on survival and can be used for improving predictive ability of existing prognostic tools in neuroblastoma patients.

\section{Material and Methods}

\section{Patients}

Seventy-one neuroblastoma patients, who were diagnosed and treated between January 2002 and December 2016, were retrospectively evaluated. All patients were staged according to International Neuroblastoma Staging System (INSS). ${ }^{14}$ Patients were treated according to national neuroblastoma protocols of Turkish Pediatric Oncology Group (TPOG); TPOG-Neuroblastoma 2003, and TPOGNeuroblastoma 2009 protocols. The poor prognostic factors are older age at diagnosis [ $\geq 18$ months for TPOG- 2009 and $\geq 12$ months for TPOG 2003], advanced stage [stage 3-4], unfavorable histology, presence of genetic alterations (MYCN amplification, chromosome $1 p, 11 q$ and/or $17 q$ deletion). Being stage IV and older than 18 months for TPOG- 2009 and $\geq 12$ months for TPOG 2003 made patients a highrisk group regardless of MYCN amplification and histology in both protocols. According to these parameters patients were stratified in to three risk groups: high, intermediate and low risk. Data including age, gender, clinical findings, stage, histopathology, genetic alterations, treatment modalities, survival of patients, peripheral blood MP and LMR were retrospectively analyzed from the files. The peripheral MP and LMR were determined from routine complete blood counts with five-part differential counts (absolute and percent of lymphocytes, monocytes, eosinophils, basophils and neutrophils) obtained at diagnosis using ADVIA 2120 Hematology System (Siemens, NY, USA). Peripheral MP was calculated by dividing the absolute monocyte count to total leukocyte count and multiplying by 100 . Lymphocyte to monocyte ratio was calculated as dividing the absolute lymphocyte count to absolute monocyte count (ALC/AMC).

After obtaining the ethics approval from the local ethics committee (Akdeniz University KAEK -2020-735), the study was initiated with informed consent from the patients.

\section{Statistical analysis}

Statistical analyses were calculated by SPSS (version 20.0) software program. The choice of the best cutoff values of peripheral MP and the LMR for assessing survival was based on their utility as a marker for the clinically relevant binary outcome of death/survival using the receiver operating characteristics curves (ROC) and area under the curve (AUC). Chi-square test was used to determine relationships between categorical variables and Mann Whitney U test was used to compare the continuous variables related to two groups. Overall survival (OS) and event-free survival (EFS) were analyzed using the approach of Kaplan-Meier. Differences between survival curves were tested for statistical significance using the two-tailed logrank test. The stepwise (backward selection) Cox proportional hazard model was used for the univariate and multivariate analyses to evaluate the variables under the prognostic factors' section to assess their impact on overall survival and event free survival. All $p$ values are two-tailed and $p$ values less than 0.05 were considered statistically significant. 
Table I. Characteristics of patients.

\begin{tabular}{|c|c|}
\hline Age at diagnosis (mean, months) & $31.2(1-204)$ \\
\hline Gender & $\mathrm{N}(\%)$ \\
\hline Female & $38(53.5)$ \\
\hline Male & $33(46.5)$ \\
\hline \multicolumn{2}{|l|}{ Age at diagnosis } \\
\hline$<12$ months & $17(24.9)$ \\
\hline$\geq 12$ months & $54(76.1)$ \\
\hline \multicolumn{2}{|l|}{ Stage } \\
\hline Stage I & $5(7)$ \\
\hline Stage II & $5(7)$ \\
\hline Stage III & $13(18.3)$ \\
\hline Stage IV & $40(56.4)$ \\
\hline Stage IVS & 8 (11.3) \\
\hline \multicolumn{2}{|l|}{ Histology } \\
\hline Favorable & $18(25.4)$ \\
\hline Unfavorable & $42(59.2)$ \\
\hline NA & $11(15.5)$ \\
\hline \multicolumn{2}{|l|}{ Chromosome $1 \mathrm{p} 11 \mathrm{q}$ deletion } \\
\hline Presence & $5(7)$ \\
\hline Absence & $5(7)$ \\
\hline Not determined & $61(86)$ \\
\hline \multicolumn{2}{|l|}{ Risk group } \\
\hline Low & $11(15)$ \\
\hline Intermediate & $15(21)$ \\
\hline High & $45(64)$ \\
\hline \multicolumn{2}{|l|}{ MYCN amplification } \\
\hline High & $19(41.3)$ \\
\hline Normal & $27(58.7)$ \\
\hline Not determined & $25(35.2)$ \\
\hline \multicolumn{2}{|l|}{ Chemotherapy } \\
\hline Yes & $62(87.3)$ \\
\hline No & $9(12.7)$ \\
\hline \multicolumn{2}{|l|}{ Surgery } \\
\hline At diagnosis & $19(26.7)$ \\
\hline Second look & $29(40.8)$ \\
\hline No & $23(32.5)$ \\
\hline \multicolumn{2}{|l|}{ Radiotherapy } \\
\hline Yes & $22(31)$ \\
\hline No & $49(69)$ \\
\hline \multicolumn{2}{|l|}{ Autologous transplantation } \\
\hline Yes & $25(35)$ \\
\hline No & $46(65)$ \\
\hline \multicolumn{2}{|l|}{ Relapsed } \\
\hline Yes & $22(31)$ \\
\hline No & $49(69)$ \\
\hline
\end{tabular}

Table I. Continued.

\begin{tabular}{lc}
\hline Cellular status at diagnosis & \\
Mean leukocyte count $/ \mathrm{mm}^{3}$ & $8918(2070-20100)$ \\
Mean absolute lymphocyte & $3143(300-12320)$ \\
Count (ALC) $/ \mathrm{mm}^{3}$ & \\
Mean absolute monocyte & $715(100-2300)$ \\
Count (AMC) $/ \mathrm{mm}^{3}$ & \\
Monocyte percentage (MP) $\%$ & $8.2(2-22)$ \\
Mean ALC/AMC (LMR) & $5(1-16)$ \\
\hline
\end{tabular}

\section{Results}

\section{Clinical Characteristics}

Totally 71 neuroblastoma patients were enrolled. Thirty-eight $(53.5 \%)$ of the patients were female and the mean age at diagnosis was 31.2 months (range; 1-204 months). Patients were staged according to INSS; 5 patients had (7\%) stage I, $5(7 \%)$ patients had stage II, $13(18.3 \%)$ patients had stage III, $40(56.4 \%)$ patients had stage IV and $8(11.3 \%)$ patients had stage IVS disease. Metastasis was detected in 48 patients $(67.6 \%)$ at initial diagnosis. MYCN amplification were high in $19(41.3 \%)$ of patients and non-amplified in $27(58.7 \%)$ of patients. MYCN status were unknown in 25 (35.2\%) patients. Eleven of them were $\geq 18$ months (or $\geq 12$ months) and had stage IV, 7 patients were $\geq 18$ months (or $\geq 12$ months) and had unfavorable histology, 7 patients had stage I -II disease. Forty-five patients (64\%) were in high-risk group, 15 patients (21\%) were in intermediate risk group and $11(15 \%)$ patients were in low-risk group. Fifteen of patients with unknown MYCN status in high-risk group, while the others were in low-risk group. Clinical characteristics of patients are shown in (Table I).

At diagnosis, mean leukocyte count was 8918/ $\mathrm{mm}^{3}$ (range; 2070-20100), mean peripheral absolute lymphocyte count (ALC) was 3143/ $\mathrm{mm}^{3}$ (range; 300-12320), mean peripheral absolute monocyte count (AMC) was $715 / \mathrm{mm}^{3}$ (range; 100-2300), mean MP was $8.2 \%$ (range; $2 \%-22 \%$ ) and mean peripheral LMR was 5 (range; 1-16). 


\section{LMR and MP at diagnosis}

Cut-off values for LMR and peripheral blood MP were determined according to ROC analysis. Peripheral MP of $7.5 \%$ or more had an AUC of 0.74 [95\% confidence interval (CI), 0.63 to 0.86 ] with a sensitivity of $73 \%$ and specificity of $70 \%$ (Fig. 1). A LMR of 3.5 or less had an AUC of 0.75 ( $95 \%$ CI, 0.64 to 0.86 ) with a sensitivity of $80 \%$ and a specificity of $61 \%$ (Fig. 2). Area under the curve values from ROC analysis support the use of LMR of $\leq 3.5$ and peripheral blood MP $\geq 7.5 \%$ as the cut-off values as markers of binary clinical outcome of survival. Patients were then assigned either to the high LMR (LMR > 3.5) group and low LMR (LMR $\leq 3.5)$ group. Forty patients were in high LMR group and 31 patients were in low LMR group. According to cut-off value of MP, 39 patients were in high MP (MP $\geq 7.5), 32$ patients were in low peripheral MP (MP <7.5) group.

When the patients were classified according to peripheral MP levels, there were more patients in the high peripheral MP group with metastatic disease $(p=0.005)$, stage 4 disease $(\mathrm{p}<0.001)$, unfavorable histology $(\mathrm{p}<0,001)$, MYCN amplification ( $\mathrm{p}=0.003)$, high risk disease $(p<0.001)$, and older age ( $\geq 12$ months; $\mathrm{p}=0.015$ ) compared to the low peripheral MP group. Also, in the low LMR group there were more patients with stage 4 disease $(p=0.014)$ unfavorable histology $(\mathrm{p}=0,046)$ and high-risk disease $(p=0.003)$ (Table II).

\section{Outcome:}

The median follow-up time after diagnosis was 22 months (range; 1-182 months). The 3 year OS and EFS rates were $51 \% \pm 6.0$ and $37 \% \pm 6.1$, respectively. The 3 year OS and EFS rates in low LMR group were significantly lower than that in the high LMR group (3-year OS: $38 \% \pm 8.8$ vs $62 \% \pm 7.8 \mathrm{p}=0.002$ and 3-year EFS: $22 \% \pm 7.7$ vs $50 \% \pm 8.5 p=0.003$ ) (Fig. 3). The patients with high peripheral MP had significantly lower 3-year OS and EFS compared to the patients with low peripheral MP (3-year OS: $38.5 \% \pm 7.8$ vs $67.5 \% \pm 8.5, \mathrm{p}=0.005$ and 3-year EFS: $24 \% \pm 7.1$ vs $54 \% \pm 9.5 \mathrm{p}=0.027$ ) (Fig. 3).
The univariate analysis showed that stage IV disease, unfavorable histology, high risk disease, low LMR and high MP were associated with low EFS and OS (Table III). The multivariate analysis revealed that low LMR (Hazard ratio

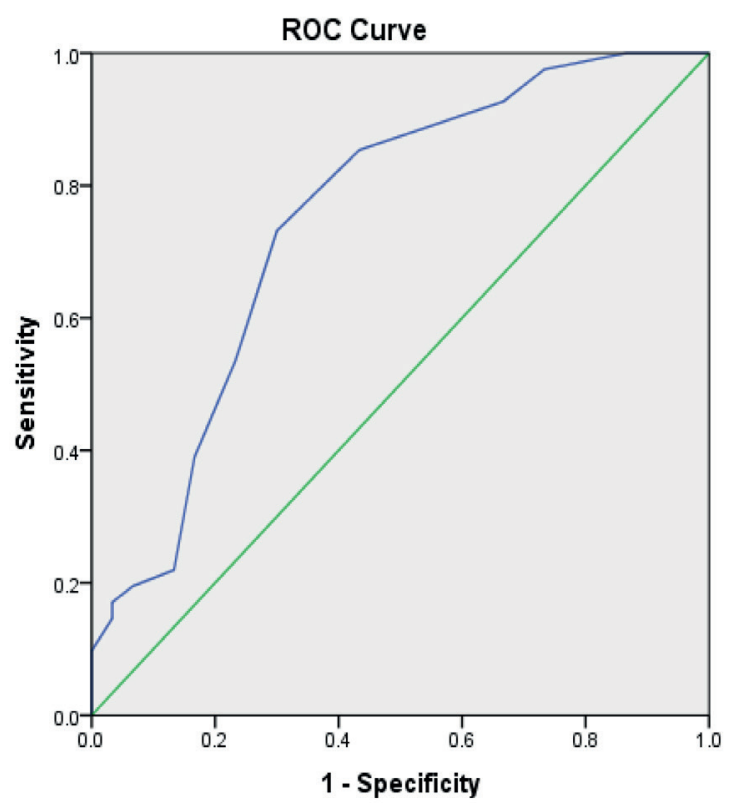

Fig. 1. Monocyte percentage of $\geq 7.5 \%$ had an AUC of 0.74 [95\% confidence interval (CI), 0.63 to 0.86 ] with a sensitivity of $73 \%$ and specifity of $70 \%$.

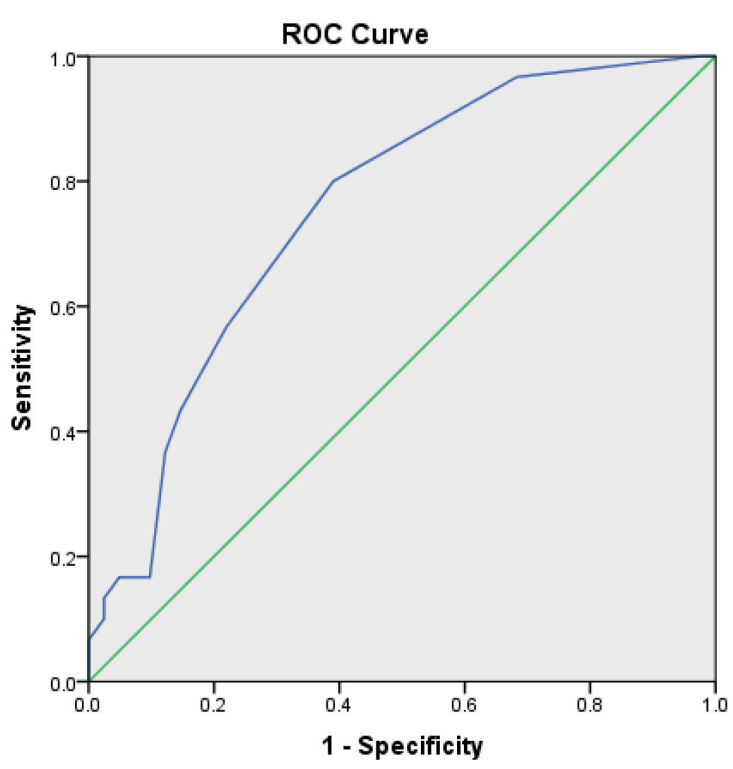

Fig. 2. Lymphocyte monocyte ratio of $\leq 3.5$ had an AUC of 0.75 [95\% confidence interval (CI), 0.64 to 0.86 ] with a sensitivity of $80 \%$ and specifity of $61 \%$. 
Table II. Characteristics of patients according to LMR and MP.

\begin{tabular}{|c|c|c|c|c|c|c|}
\hline \multirow[b]{2}{*}{ Variables } & \multicolumn{2}{|c|}{ LMR } & \multirow[b]{2}{*}{ p-value } & \multicolumn{2}{|c|}{ MP } & \multirow[b]{2}{*}{$\mathrm{p}$-value } \\
\hline & $\begin{array}{l}\text { High }(>3.5) \\
\quad(n=40)\end{array}$ & $\begin{array}{c}\text { Low }(\leq 3.5) \\
(n=31)\end{array}$ & & $\begin{array}{l}\text { High }(\geq 7.5 \%) \\
\qquad(n=39)\end{array}$ & $\begin{array}{c}\text { Low }(<7.5 \%) \\
\quad(n=32)\end{array}$ & \\
\hline \multicolumn{7}{|l|}{ Age at diagnosis } \\
\hline$<12$ months & $13(32.5)$ & 4 (12.9) & 0.055 & $5(12.8)$ & $12(37.5)$ & 0.015 \\
\hline$\geq 12$ months & $27(67.5)$ & $27(87.1)$ & & $34(87.2)$ & $20(62.5)$ & \\
\hline \multicolumn{7}{|l|}{ Gender, N (\%) } \\
\hline Female & $20(50)$ & $18(58.1)$ & 0.499 & $20(51.3)$ & $18(56.2)$ & 0.676 \\
\hline Male & $20(50)$ & $13(41.9)$ & & $19(48.7)$ & $14(43.8)$ & \\
\hline \multicolumn{7}{|l|}{ Stage, N (\%) } \\
\hline Stage $1,2,3,4 S$ & $22(55.0)$ & $8(25.8)$ & 0.014 & $8(20.5)$ & $22(68.8)$ & $<0.001$ \\
\hline Stage 4 & $18(45.0)$ & $23(74.2)$ & & $31(79.5)$ & $10(31.3)$ & \\
\hline \multicolumn{7}{|l|}{ Metastatic Disease } \\
\hline Presence & 25 & 23 & 0.29 & 32 & 16 & 0.005 \\
\hline Absence & 15 & 8 & & 7 & 16 & \\
\hline \multicolumn{7}{|l|}{ Risk Group, N (\%) } \\
\hline Low-Intermediate & $20(50.0)$ & $5(16.1)$ & 0.003 & $5(12.8)$ & $20(62.5)$ & $<0.001$ \\
\hline High & $20(50.0)$ & $26(83.9)$ & & $34(87.2)$ & $12(37.5)$ & \\
\hline \multicolumn{7}{|l|}{ Histology, N (\%) } \\
\hline Favorable & $14(40.0)$ & $4(16.0)$ & 0.046 & $2(6.7)$ & $16(53.3)$ & $<0.001$ \\
\hline Unfavorable & $21(60.0)$ & $21(84.0)$ & & $28(93.3)$ & $14(46.7)$ & \\
\hline \multicolumn{7}{|l|}{ MYCN amplification } \\
\hline High & 7 (29.2) & $12(54.5)$ & 0.081 & $16(59.3)$ & $3(15.8)$ & 0.003 \\
\hline Normal & $17(70.8)$ & $10(45.5)$ & & $11(40.7)$ & $16(84.2)$ & \\
\hline
\end{tabular}

(HR), 2.29; 95\% CI, 1.11-4.75; $\mathrm{p}=0.025)$ and stage IV disease (HR, 2.97; 95\% CI, 1.26-7.02; $\mathrm{p}=0.013$ ) were the factors that were significantly associated with low 3-year OS. Low LMR (HR, 2.15; 95\% CI, 1,07-4.30; $\mathrm{p}=0.03)$ and stage IV disease (HR, 3.13; 95\% CI, 1.40-7.02; $\mathrm{p}=0.006$ ) were also defined as independent factors for decreased 3-year EFS (Table III).

\section{Discussion}

Our study has shown that a low LMR might be a poor prognostic factor in pediatric neuroblastoma patients. In addition, a high MP was also associated with low EFS and OS even though the noteworthy association between high MP and clinical outcome could not be established in the multivariate analysis. Current literature has indicated that the combination of cellular components of the systemic inflammatory response, such as monocyte count, L/M ratio, and MP represent significant markers of clinical outcome in a wide variety of cancers. ${ }^{15,16}$ Lymphocytes are regarded as the crucial factors in immune surveillance, and the presence of an immunologic antitumor reaction is based on lymphocytic infiltration into the tumor microenvironment. ${ }^{17,18}$ The prognostic role of LMR was first described by Porrata et al. ${ }^{6}$ in Hodgkin Lymphoma patients in 2011. Shortly after this study, in 2012, Li et al. ${ }^{7}$ documented that LMR was also an independent prognostic factor of survival in diffuse large B-cell lymphoma patients. The association between low LMR and poor OS was shown later on in non-hematologic solid tumors. Li et al. ${ }^{19}$ documented that pretreatment LMR level was a significant favorable factor for prediction of the clinical outcome in nasopharyngeal carcinoma patients. The following reports were similar 


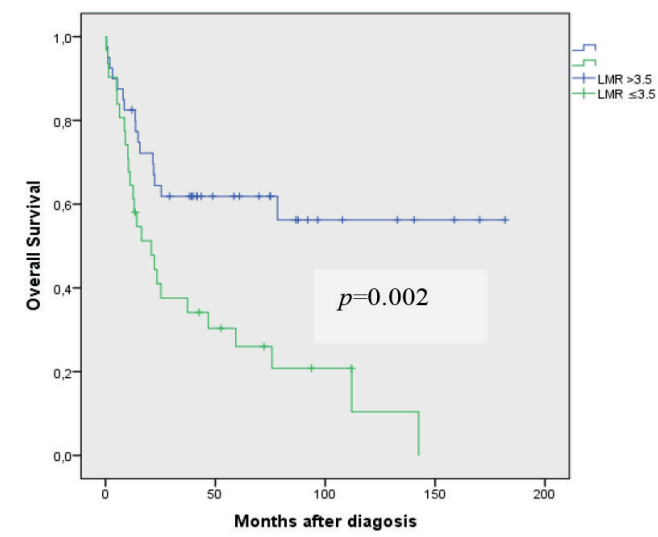

C

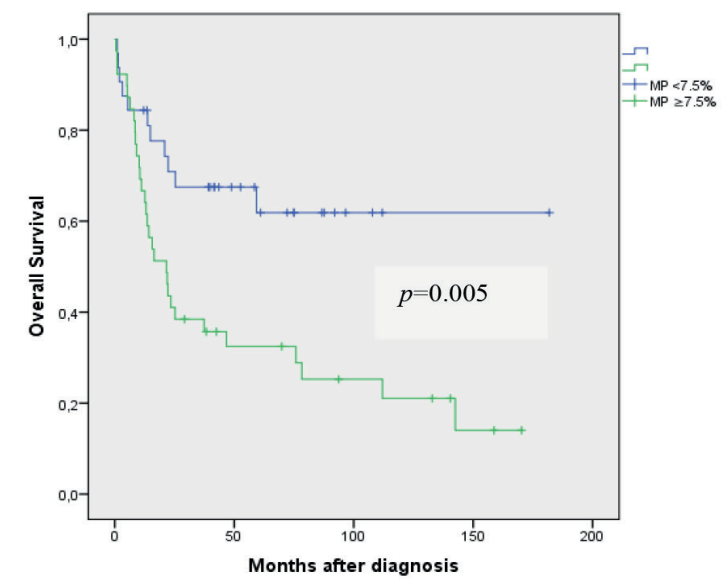

B

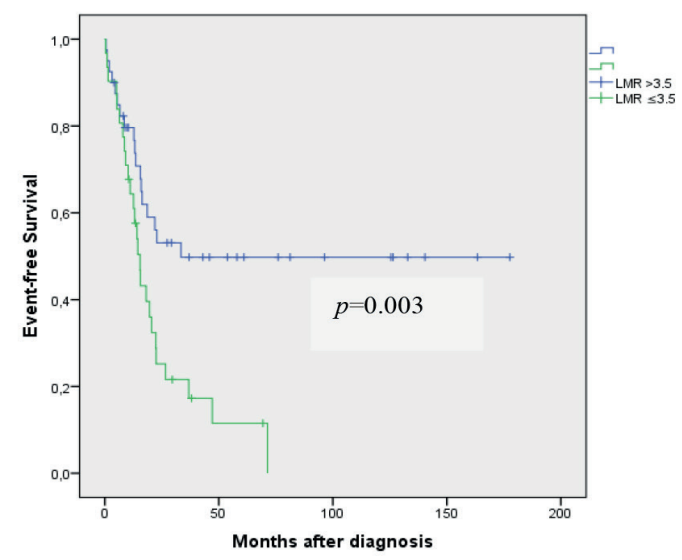

$\mathrm{D}$

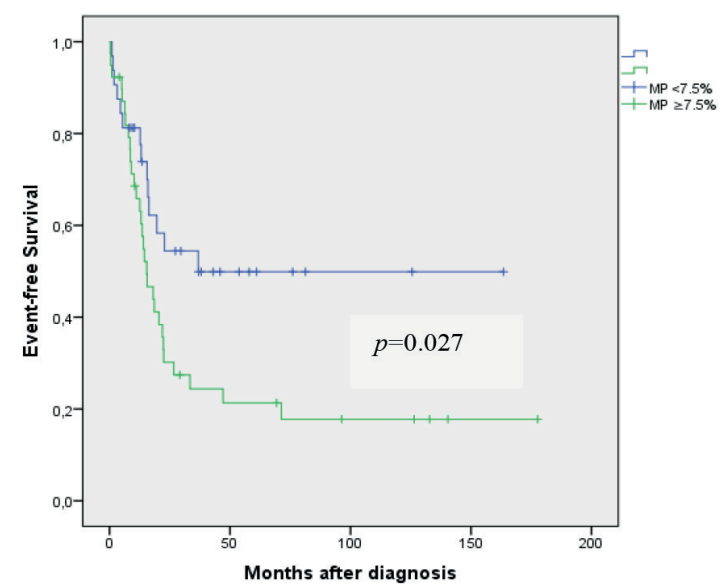

Fig. 3. Comparison of low and high LMR on OS (A), EFS (B) and low and high MP on OS (C) and EFS (D). EFS: event-free survival, OS: overall survival, LMR: lymphocyte to monocyte ratio, MP: blood monocyte percentage

Table III. Univariate and multivariate analyses for OS and EFS.

\begin{tabular}{lcccccc}
\hline Covariate & \multicolumn{3}{c}{ OS } & & EFS & \\
\hline Univariate analysis & HR & $95 \%$ CI & p-value & HR & $95 \%$ CI & p-value \\
$\quad$ LMR $\leq 3.5$ & 2.65 & $1.41-4.99$ & 0.002 & 2.48 & $1.34-4.56$ & 0.004 \\
MP $\geq 7.5 \%$ & 2.60 & $1.30-5.21$ & 0.007 & 2.02 & $1.07-3.81$ & 0.030 \\
$\quad$ Stage 4 disease & 3.13 & $1.49-6.57$ & 0.003 & 3.13 & $1.54-6.35$ & 0.002 \\
$\quad$ Unfavorable histology & 3.77 & $1.32-10.74$ & 0.013 & 4.25 & $1.5-12.06$ & 0.006 \\
$\quad$ High risk & 2.84 & $1.25-6.42$ & 0.012 & 2.74 & $1.27-5.92$ & 0.010 \\
Multivariate analysis & & & & & & \\
$\quad$ LMR $\leq 3.5$ & 2.29 & $1.11-4.75$ & 0.025 & 2.15 & $1.07-4.30$ & 0.030 \\
$\quad$ Stage 4 disease & 2.97 & $1.26-7.02$ & 0.013 & 3.13 & $1.40-7.02$ & 0.006 \\
\hline
\end{tabular}

LMR: lymphocyte to monocyte ratio

MR: monocyte percentage 
to previously reported data; a lower LMR was associated with lymph nodes metastasis, tumor progression and poor 5-year cancer specific survival in esophageal squamous cell carcinoma patients. ${ }^{20}$ The low LMR was related with more aggressive tumor behavior and worse long-term survival in resectable gastric adenocarcinoma patients. ${ }^{21}$ Low LMR was significantly correlated with higher degree of tumor infiltration and poor prognosis also in other digestive system cancers such as pancreas and colorectal carcinomas. ${ }^{22,23}$ Deng et al. ${ }^{24}$ have retrospectively evaluated 317 newly diagnosed locally advanced rectal cancer patients and shown that the LMR was a valuable prognostic factor for predicting the OS in this group of patients. A meta-analysis showed that the patients with lower LMR had poorer OS in non-small cell lung cancer. ${ }^{25}$ Low LMR was also associated with unfavorable survival in patients with ovarian cancer and could serve as a prognostic biomarker. ${ }^{26}$

The definite mechanism of the association between low LMR and poor survival of cancer patients are not totally explained. This relevance may de described through tumor infiltrating immune cells which contribute significantly in destruction or development of tumor growth. In tumor microenvironment the lymphocytes are considered as one of the most vital components of the host's cellular immunity. Therefore, a low lymphocyte count might be responsible for a fragile, inadequate reaction to tumor and thereby an exacerbated clinical outcome. ${ }^{17}$

Macrophage is another essential component of tumor infiltrating inflammatory cells. Tumor associated macrophages (TAM) are derived from peripheral blood monocytes and a positive correlation between TAM and peripheral blood monocyte count has been shown in previous studies. ${ }^{27,28}$ Therefore, circulating level of monocytes may reflect formation or presence of TAMs. TAM may promote angiogenesis, metastasis, immune suppression and chemo resistance. ${ }^{5,29-31}$ Macrophages do not only contribute to tumor growth but also impair effective anti-tumor lymphocyte response. Some clinical studies have indicated a significant correlation between elevated macrophage content and poor clinical outcome in soft tissue cancer patients. ${ }^{32}$

Metastatic disease at diagnosis is one of the most important prognostic factors for neuroblastoma. ${ }^{10}$ It was shown that TAM enhance neo-angiogenesis, promote tumor cell migration and metastases. ${ }^{27,30,33}$ The high peripheral monocyte count can reflect the presence or formation of TAM and has been reported as a poor prognostic factor in adult patients with various types of cancer. ${ }^{33}$ Koh et $a .^{27}$ also reported that there was a positive correlation between TAM and MP in patients with Hodgkin lymphoma. We found that there were more patients with advanced stage and metastasis in the group who had high MP, unfortunately we could not evaluate tumor microenvironment. There is a unique study by Asgharzadeh et al. ${ }^{34}$ which shows significantly greater numbers of infiltrating macrophages in tumor samples of neuroblastoma patients with metastatic (stage 4) disease. These findings support that number of peripheral monocyte count, which will convert to TAM, may have prognostic significance in neuroblastoma. However, there was a prognostic significance of high MP in neuroblastoma outcome according to univariate analysis, multivariate analysis did not reveal that high MP was an independent prognostic factor for EFS and OS in our study. Further studies including higher number of patients are needed to determine the prognostic value of peripheral monocytes count or MP in neuroblastoma patients.

The limitations of this study are the retrospective nature of the design, small number of patients, short follow up period and absence of tumor microenvironment evaluation. We evaluated LMR and MP on outcome of neuroblastoma patients. LMR was found as an independent prognostic factor for EFS and OS in this study. The peripheral blood count and cell count ratio can be determined readily and inexpensively by a standard automated complete blood count machine. LMR and MP may be new 
prognostic factors for neuroblastoma and can be used for predicting survival which is a cost effective and easy-accessible biomarker. Our results should be verified by multi-centric and prospective studies which also evaluate the tumor microenvironment concomitantly.

\section{Acknowledgement}

The authors would like to thank all doctors and medical staff involved in medical care of neuroblastoma patients for their exceptional work.

\section{Author contribution}

The authors confirm contribution to the paper as follows: study conception and design: KY, EG; data collection: $\mathrm{KY}, \mathrm{AK}$; analysis and interpretation of results: SB, GT, AK, EG; draft manuscript preparation: KY, GT, FTK, EG; All authors reviewed the results and approved the final version of the manuscript.

\section{Ethical approval}

After obtaining the ethics approval from the local ethics committee (Akdeniz University KAEK -2020-735), the study was initiated with informed consent from the patients

\section{Source of funding}

We have nothing to disclose

\section{Conflict of interest}

All the authors certify that they have no affiliations with or involvement in any organization or entity with any financial interest or non-financial interest in the subject matter or materials discussed in this manuscript.

\section{REFERENCES}

1. Tredan O, Manuel M, Clapisson G, et al. Patients with metastatic breast cancer leading to CD4+ T cell lymphopaenia have poor outcome. Eur J Cancer 2013; 49: 1673-1682.

2. Liang $\mathrm{L}$, Zhu J, Jia $\mathrm{H}$, et al. Predictive value of pretreatment lymphocyte count in stage II colorectal cancer and in high-risk patients treated with adjuvant chemotherapy. Oncotarget 2016; 7: 10141028.

3. Milne $\mathrm{K}$, Alexander $\mathrm{C}$, Webb JR, et al. Absolute lymphocyte count is associated with survival in ovarian cancer independent of tumor-infiltrating lymphocytes. J Transl Med 2012; 10: 33.

4. Mehrazin R, Uzzo RG, Kutikov A, et al. Lymphopenia is an independent predictor of inferior outcome in papillary renal cell carcinoma. Urol Oncol 2015; 33: 388.e19-388.e.25.

5. Jinushi M, Komohara Y. Tumor-associated macrophages as an emerging target against tumors: creating a new path from bench to bedside. Biochim Biophys Acta 2015; 1855: 123-130.

6. Porrata LF, Ristow K, Colgan JP, et al. Peripheral blood lymphocyte/monocyte ratio at diagnosis and survival in classical Hodgkin's lymphoma. Haematologica 2012; 97: 262-269.

7. Li ZM, Huang JJ, Xia Y, et al. Blood lymphocyte-tomonocyte ratio identifies high-risk patients in diffuse large B-cell lymphoma treated with R-CHOP. PLoS One 2012; 7: e41658.

8. Colon NC, Chung DH. Neuroblastoma. Adv Pediatr 2011; 58: 297-311.

9. Birch JM, Blair V. The epidemiology of infant cancers. Br J Cancer Suppl 1992; 18: S2-S4.

10. Irwin MS, Park JR. Neuroblastoma: paradigm for precision medicine. Pediatr Clin North Am 2015; 62: 225-256.

11. Yamamoto K, Hanada R, Kikuchi A, et al Spontaneous regression of localized neuroblastoma detected by mass screening. J Clin Oncol 1998; 16: 1265-1269.

12. Cole WH, Everson TC. Spontaneous regression of cancer: preliminary report. Ann Surg 1956; 144: 366383.

13. Papac RJ. Spontaneous regression of cancer: possible mechanisms. In Vivo 1998; 12: 571-578. 
14. Brodeur GM, Pritchard J, Berthold F, et al. Revisions of the international criteria for neuroblastoma diagnosis, staging, and response to treatment. J Clin Oncol 1993; 11: 1466-1477.

15. Mantovani A, Allavena P, Sica A, Balkwill F. Cancerrelated inflammation. Nature 2008; 454: 436-444.

16. Chan JC, Chan DL, Diakos CI, et al. The lymphocyteto-monocyte ratio is a superior predictor of overall survival in comparison to established biomarkers of resectable colorectal cancer. Ann Surg 2017; 265: 539-546.

17. Hoffmann TK, Dworacki G, Tsukihiro T, et al. Spontaneous apoptosis of circulating $\mathrm{T}$ lymphocytes in patients with head and neck cancer and its clinical importance. Clin Cancer Res 2002; 8: 2553-2562.

18. Dunn GP, Old LJ, Schreiber RD. The immunobiology of cancer immunosurveillance and immunoediting. Immunity 2004; 21: 137-148.

19. Li J, Jiang R, Liu WS, et al. A large cohort study reveals the association of elevated peripheral blood lymphocyte-to-monocyte ratio with favorable prognosis in nasopharyngeal carcinoma PLoS One 2013; 8: e83069.

20. Huang Y, Feng JF. Low preoperative lymphocyte to monocyte ratio predicts poor cancer-specific survival in patients with esophageal squamous cell carcinoma. Onco Targets Ther 2015; 8: 137-145.

21. Hsu JT, Wang CC, Le PH, et al. Lymphocyte-tomonocyte ratios predict gastric cancer surgical outcomes. J Surg Res 2016; 202: 284-290.

22. Qi Q, Geng Y, Sun M, Wang P, Chen Z. Clinical implications of systemic inflammatory response markers as independent prognostic factors for advanced pancreatic cancer. Pancreatology 2015; 15: 145-150.

23. Neal CP, Cairns V, Jones MJ, et al. Prognostic performance of inflammation-based prognostic indices in patients with resectable colorectal liver metastases. Med Oncol 2015; 32: 144.

24. Deng YX, Lin JZ, Peng JH, et al. Lymphocyteto-monocyte ratio before chemoradiotherapy represents a prognostic predictor for locally advanced rectal cancer. Onco Targets Ther 2017; 10 : 5575-5583.
25. Wang Y, Huang D, Xu WY, Wang YW, Che GW. Prognostic value of pretreatment lymphocyte-tomonocyte ratio in non-small cell lung cancer: a metaanalysis. Oncol Res Treat 2019; 42: 523-531.

26. Lu C, Zhou L, Ouyang J, Yang H. Prognostic value of lymphocyte-to-monocyte ratio in ovarian cancer: a meta-analysis. Medicine (Baltimore) 2019; 98: e15876.

27. Koh YW, Shin SJ, Park C, Yoon DH, Suh C, Huh J. Absolute monocyte count predicts overall survival in mantle cell lymphomas: correlation with tumourassociated macrophages. Hematol Oncol 2014; 32: 178-186.

28. Mantovani A, Sica A, Locati M. New vistas on macrophage differentiation and activation. Eur J Immunol 2007; 37: 14-16.

29. Kitamura T, Qian BZ, Pollard JW. Immune cell promotion of metastasis. Nat Rev Immunol 2015; 15: 73-86.

30. Qian BZ, Pollard JW. Macrophage diversity enhances tumor progression and metastasis. Cell 2010; 141: 39-51.

31. Allavena P, Sica A, Garlanda C, Mantovani A. The yin-yang of tumor-associated macrophages in neoplastic progression and immune surveillance. Immunol Rev 2008; 222: 155-161.

32. Raj SKS, Kooshki M, Winters M, et al. Prognostic implications of tumor associated macrophages (TAMs) in soft tissue sarcoma. J Clin Oncol 2019; 37(Suppl 15): e22548.

33. Schmidt H, Bastholt L, Geertsen P, et al. Elevated neutrophil and monocyte counts in peripheral blood are associated with poor survival in patients with metastatic melanoma: a prognostic model. Br J Cancer 2005; 93: 273-278.

34. Asgharzadeh S, Salo JA, Ji L, et al. Clinical significance of tumor-associated inflammatory cells in metastatic neuroblastoma. J Clin Oncol 2012; 30: 3525-3532. 